\title{
Risk Factors in and Long-Term Survival of Patients with Post-Transplantation Diabetes Mellitus: A Retrospective Cohort Study
}

\author{
Ching-Yao Cheng ${ }^{1,2}\left(\mathbb{D}\right.$, Cheng-Hsu Chen ${ }^{3,4,5}$, Ming-Fen $\mathrm{Wu}^{1}$, Ming-Ju Wu $\left.{ }^{3,6,7,8} \mathbb{(}\right)$, \\ Jun-Peng Chen ${ }^{9}$, Ying-Mei Liu ${ }^{1}$, Yu-Chi Hou ${ }^{2}$ and Hue-Yu Wang ${ }^{10,11, *}$ \\ 1 Department of Pharmacy, Taichung Veterans General Hospital, Taichung 40705, Taiwan; \\ chingyao4937b@gmail.com (C.-Y.C.); mfenwu@vghtc.gov.tw (M.-F.W.); lym0361@gmail.com (Y.-M.L.) \\ 2 School of Pharmacy, China Medical University, Taichung 40402, Taiwan; hou5133@gmail.com \\ 3 Division of Nephrology, Department of Internal Medicine, Taichung Veterans General Hospital, \\ Taichung 40705, Taiwan; cschen@vghtc.gov.tw (C.-H.C.); wmj530@gmail.com (M.-J.W.) \\ 4 Department of Life Science, Tunghai University, Taichung 40704, Taiwan \\ 5 School of Medicine, College of Medicine, China Medical University, Taichung 40402, Taiwan \\ 6 School of Medicine, Chung-Shan Medical University, Taichung 40402, Taiwan \\ 7 Graduate Institute of Clinical Medical Science, College of Medicine, China Medical University, \\ Taichung 40402, Taiwan \\ 8 Graduate Institute of Biomedical Science, National Chung Hsing University, Taichung 40227, Taiwan \\ 9 Biostatistics Task Force, Taichung Veterans General Hospital, Taichung 40705, Taiwan; \\ pippan7676@vghtc.gov.tw \\ 10 Department of Pharmacy, Chi Mei Medical Center, Tainan City 71004, Taiwan \\ 11 Department of Pharmacy, Chia Nan University of Pharmacy and Science, Tainan City 71710, Taiwan \\ * Correspondence: s6686107wang@gmail.com; Tel.: +886-6281-2811 (ext. 52943)
}

Received: 20 May 2020; Accepted: 21 June 2020; Published: 25 June 2020

\begin{abstract}
Post-transplant diabetes mellitus (PTDM) is associated with infection, cardiovascular morbidity, and mortality. A retrospective cohort study involving patients who underwent renal transplantation in a transplantation center in Taiwan from January 2000 to December 2018 was conducted to investigate the incidence and risk factors of PTDM and long-term patient and graft survival rates. High age ( $45-65$ vs. $<45$ years, adjusted odds ratio $(\mathrm{aOR})=2.90,95 \%$ confidence interval $(\mathrm{CI})=1.64-5.13, p<0.001)$, high body mass index $\left(>27 \mathrm{vs} .<24 \mathrm{~kg} / \mathrm{m}^{2}, \mathrm{aOR}=5.35,95 \% \mathrm{CI}=\right.$ $2.75-10.42, p<0.001$ ), and deceased organ donor (cadaveric vs. living, $\mathrm{aOR}=2.01,95 \% \mathrm{CI}=1.03-3.93$, $p=0.04$ ) were the three most important risk factors for the development of PTDM. The cumulative survival rate of patients and allografts was higher in patients without PTDM than in those with PTDM $(p=0.007$ and 0.041 , respectively). Concurrent use of calcineurin inhibitors and mammalian target of rapamycin inhibitors (mTORis) decreased the risk of PTDM (tacrolimus vs. tacrolimus with mTORi, $\mathrm{aOR}=0.28,95 \% \mathrm{CI}=0.14-0.55, p<0.001$ ). Investigating PTDM risk factors before and modifying immunosuppressant regimens after transplantation may effectively prevent PTDM development.
\end{abstract}

Keywords: immunosuppressant; post-transplant diabetes mellitus; risk factor; survival rate

\section{Introduction}

Post-transplant diabetes mellitus (PTDM) is a common and severe complication in patients who receive immunosuppressive agents after kidney transplantation, and this has a major effect on graft and patient outcomes [1-4]. PTDM is associated with not only increased mortality and morbidity, but also increased rates of cardiovascular diseases and infections, which are the leading causes of 
death in kidney transplant recipients [5]. The incidence of PTDM is $9.1-45.3 \%$ after 1 year [1,3,6-9], $10.0-30.0 \%$ after 3 years $[1,4]$, and $10.2-15.1 \%$ after 5 years $[10,11]$. Similar to type 2 diabetes, PTDM is characterized by insulin resistance, decompensated insulin release, hypertriglyceridemia, obesity, and hypertension [12]. However, risk factors for PTDM differ from those associated with type 2 diabetes. The common risk factors for PTDM include obesity, sedentary lifestyle, other metabolic syndromes associated with obesity, certain viral infections (e.g., hepatitis $C$ virus and cytomegalovirus), and the use of drugs with diabetogenic effects administered with post-transplantation therapy, including corticosteroids and immunosuppressive agents (e.g., tacrolimus, cyclosporine, and mammalian target of rapamycin inhibitors (mTORis)) [13]. Other immunosuppressive drugs, including azathioprine and mycophenolate mofetil (MMF), are not associated with a disruption in glucose metabolism [14]. The effect of immunosuppressive agents on graft and patient survival remains controversial, especially in Asian populations, and the incidence of and risk factors for PTDM in the Taiwanese population are unclear. Here, we aimed to determine the incidence of and risk factors for PTDM in kidney transplant recipients. Furthermore, the prognosis of PTDM was investigated to determine the difference in graft and patient survival rates between patients with PTDM and those without PTDM.

\section{Patients and Methods}

A retrospective cohort study was conducted in a transplantation center in central Taiwan using electronic medical records. Patients with PTDM were defined as those with a fasting blood glucose level of $\geq 140 \mathrm{mg} / \mathrm{dL}$ recorded on at least two consecutive measurements, or those who required the administration of an oral anti-diabetic drug or insulin for $\geq 3$ months, as described in the World Health Organization/American Diabetes Association international consensus guidelines [7,8], recommendations, and future directions on PTDM [15]. Patients with temporary hyperglycemia after transplantation (e.g., administration of anti-diabetic drug or insulin for $<3$ months or corticosteroid pulse therapy for acute rejection treatment, leading to temporary hyperglycemia) were classified as patients without PTDM. The review board of Taichung Veterans General Hospital approved the study protocol (CE18303B), and the study was carried out in accordance with the Declaration of Helsinki. The need for patient consent was waived owing to the use of a decoded database in the hospital information system.

\subsection{Study Population}

This was a single-center, analytical, retrospective study involving adults ( $\geq 20$ years old) who underwent a kidney transplant between January 2000 and December 2018 and survived with a functioning allograft for $\geq 1$ year after transplantation. Recipients with more than one organ transplant, post-transplant follow-up of $<1$ year, graft failure, and/or death in the first year were excluded. Patients with pre-transplant diabetes were also excluded (Figure 1). The primary endpoint of the study was the incidence of and risk factors for PTDM in kidney transplant recipients, and the secondary endpoint was allograft and patient survival. The end time of PTDM risk analyses was the date of PTDM diagnosis or study end.

\subsection{Immunosuppressant Regimens (IMRs)}

Post-transplantation immunosuppressant regimens, including calcineurin inhibitors (CNIs), MMF, and corticosteroids, were used, and mTORis were added as required to prevent organ rejection. Recipients were divided into four groups according to the IMRs received to analyze the correlation and hazards linking IMRs and PTDM: FK group, tacrolimus-based regimen; CsA group, cyclosporine-based regimen; FK + mTORi group, tacrolimus-based regimen combined with an mTORi; and CsA + mTORi group, cyclosporine-based regimen combined with an mTORi. Patients who were initially administered tacrolimus or cyclosporine and whose regimen was altered were excluded. 


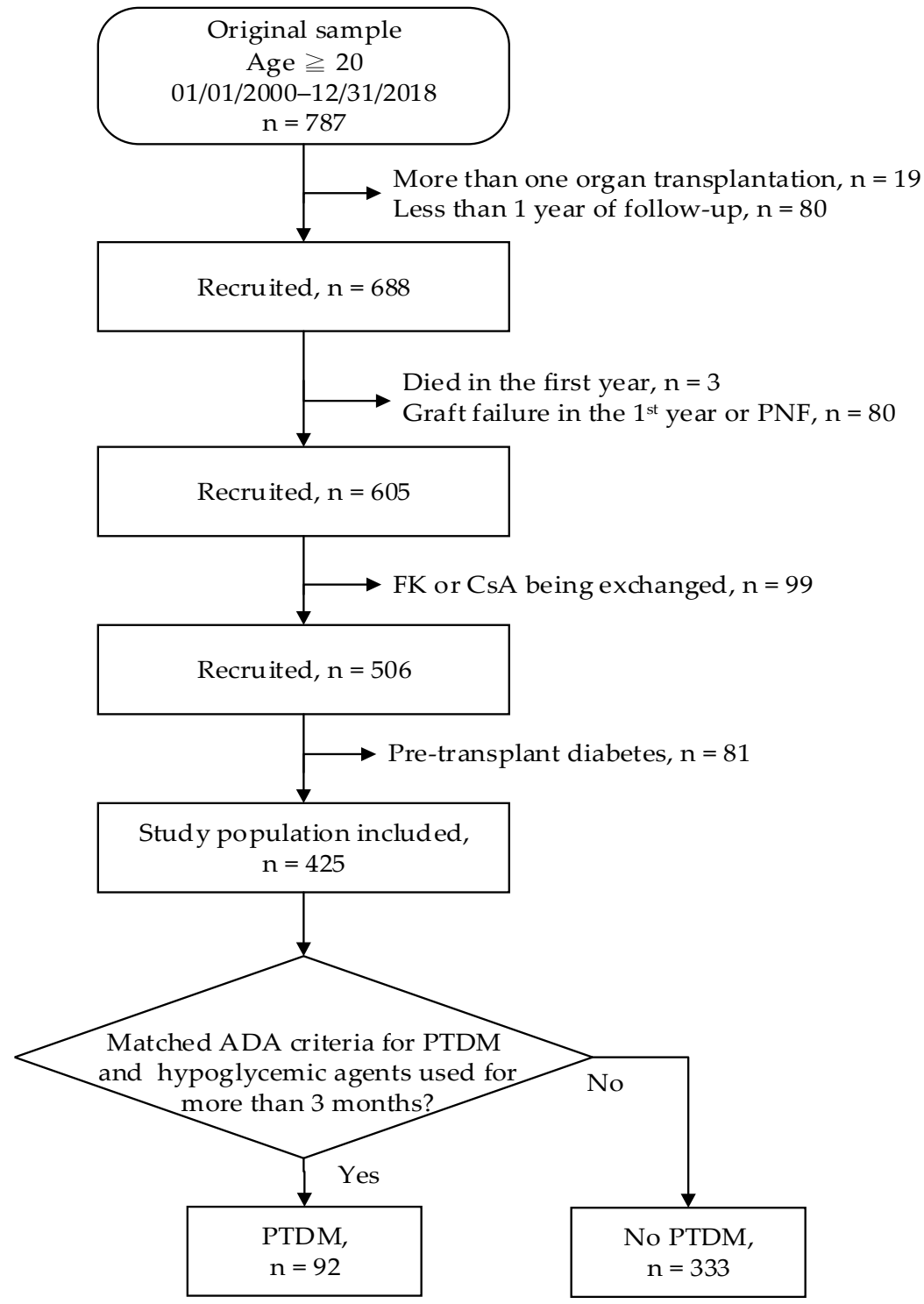

Figure 1. Flowchart of patient inclusion process. ADA: American Diabetes Association; CsA: cyclosporine; FK: tacrolimus; PNF: primary non-function; PTDM: post-transplant diabetes mellitus.

\subsection{Statistical Analysis}

Continuous variables are expressed as median (interquartile range, IQR). Mann-Whitney U and chi-square tests were used to compare continuous and categorical variables in PTDM-negative and PTDM-positive patients. Survival curves were analyzed using the Kaplan-Meier method. Independent risk factors for PTDM were identified using a stepwise forward multivariate logistic regression model. Statistical analyses were performed using SPSS version 22.0 statistical software (SPSS Company, New York, NY, USA) and $p<0.05$ indicated statistical significance.

\section{Results}

\subsection{Study Population}

A total of 787 patients underwent renal transplantation, and 425 were enrolled in this study (Figure 1). There were $221(52.0 \%)$ male and 204 (48.0\%) female patients (Table 1). The median age of the study population at transplant was $44.0(\mathrm{IQR}=34.1-51.9)$ years and the median follow-up period was $10.3(\mathrm{IQR}=6.4-13.8)$ years. 
Table 1. Characteristics of the kidney transplantation study population.

\begin{tabular}{|c|c|c|c|c|c|}
\hline \multirow{3}{*}{$\begin{array}{c}\text { Variable } \\
\text { Age at KT, years † } \\
\text { Gender }\end{array}$} & \multicolumn{2}{|c|}{ Non-PTDM $(n=333)$} & \multicolumn{2}{|c|}{$\operatorname{PTDM}(n=92)$} & \multirow{3}{*}{$\begin{array}{c}p \text {-Value } \\
<_{0.001}^{* *} \\
0.272^{*}\end{array}$} \\
\hline & 41.8 & $(33.0-50.8)$ & 49.7 & $(40.5-56.9)$ & \\
\hline & & & & & \\
\hline Female & 165 & $(49.5 \%)$ & 39 & $(42.4 \%)$ & \\
\hline Male & 168 & $(50.5 \%)$ & 53 & $(57.6 \%)$ & \\
\hline $\mathrm{BMI}+$ & 22.3 & $(20.2-24.6)$ & 24.6 & $(22.6-27.7)$ & $<0.001^{* *}$ \\
\hline Donor source & & & & & $0.004^{* *}$ \\
\hline Living & 114 & $(34.2 \%)$ & 17 & $(18.5 \%)$ & \\
\hline Cadaveric & 219 & $(65.8 \%)$ & 75 & $(81.5 \%)$ & \\
\hline Dialysis pre-transplant & & & & & 1.000 \\
\hline $\mathrm{HD} / \mathrm{PD}$ & 283 & $(85.2 \%)$ & 78 & $(84.4 \%)$ & \\
\hline pre-empty & 49 & $(14.8 \%)$ & 14 & $(15.2 \%)$ & \\
\hline Causes of patient death & & & & & $0.036^{*}$ \\
\hline Infection & 8 & $(40.0 \%)$ & 9 & $(60.0 \%)$ & \\
\hline Cancer & 11 & $(55.0 \%)$ & 1 & $(6.7 \%)$ & \\
\hline Hepatic failure & 0 & $(0.0 \%)$ & 2 & $(13.3 \%)$ & \\
\hline Cardiovascular sudden death & 0 & $(0.0 \%)$ & 1 & $(6.7 \%)$ & \\
\hline CVA & 0 & $(0.0 \%)$ & 1 & $(6.7 \%)$ & \\
\hline Others & 1 & $(5.0 \%)$ & 1 & $(6.7 \%)$ & \\
\hline \multicolumn{6}{|l|}{ Comorbidity before KT } \\
\hline Hypertension & 213 & $(64.0 \%)$ & 44 & $(47.8 \%)$ & $0.007 * *$ \\
\hline Anemia & 154 & $(46.2 \%)$ & 37 & $(40.2 \%)$ & 0.362 \\
\hline Hyperuricemia & 82 & $(24.6 \%)$ & 14 & $(15.2 \%)$ & 0.077 \\
\hline Hyperlipidemia & 75 & $(22.5 \%)$ & 17 & $(18.5 \%)$ & 0.490 \\
\hline Hepatitis B & 16 & $(4.8 \%)$ & 3 & $(3.3 \%)$ & 0.776 \\
\hline Hepatitis C & 13 & $(3.9 \%)$ & 2 & $(2.2 \%)$ & 0.540 \\
\hline Cancer & 8 & $(2.4 \%)$ & 3 & $(3.3 \%)$ & 0.710 \\
\hline MACE & 2 & $(0.6 \%)$ & 0 & $(0.0 \%)$ & 1.000 \\
\hline CMV & 3 & $(0.9 \%)$ & 1 & $(1.1 \%)$ & 1.000 \\
\hline BK viremia & 2 & $(0.6 \%)$ & 0 & $(0.0 \%)$ & 1.000 \\
\hline IMRS groups & & & & & $0.001^{* *}$ \\
\hline FK & 183 & $(55.0 \%)$ & 64 & $(69.6 \%)$ & \\
\hline $\mathrm{FK}+\mathrm{mTORi}$ & 124 & $(37.2 \%)$ & 17 & $(18.5 \%)$ & \\
\hline $\mathrm{CsA}$ & 12 & $(3.6 \%)$ & 9 & $(9.8 \%)$ & \\
\hline $\mathrm{Cs} \mathrm{A}+\mathrm{mTORi}$ & 14 & $(4.2 \%)$ & 2 & $(2.2 \%)$ & \\
\hline
\end{tabular}

Chi-square test. + Mann-Whitney U test. ${ }^{*} p<0.05,{ }^{* *} p<0.01$. PTDM: post-transplant diabetes mellitus; BMI: body mass index; CMV: cytomegalovirus; CVA: cerebrovascular accident; KT: kidney transplantation; HD/PD: hemodialysis/peritoneal dialysis; IMRs: immunosuppressive regimens; FK: tacrolimus; MACE: major adverse cardiovascular events; mTORi: mammalian target of rapamycin inhibitor; CsA: cyclosporine.

\subsection{Incidence of PTDM}

The median time to PTDM diagnosis was $2.62(\mathrm{IQR}=0.4-5.56)$ years. The frequency of PTDM after 10 years was $21.6 \%(92 / 425)$. The incidence rate was the highest in the first year after transplantation $(7.7 \%)$, followed by the second year (4.0\%). The cumulative incidence was $25.6 \%$ over 10 years (Table 2 ). There were no obvious differences with regard to gender or hemodialysis before transplantation between patients in the PTDM and non-PTDM groups (Table 1). The incidence of PTDM was significantly higher in patients with high age and body mass index (BMI; $p<0.001$ ). Among the 92 patients who developed PTDM, 75 received renal transplants from deceased donors, demonstrating significant differences in the development of PTDM among patients receiving organs from different donor sources $(p=0.004)$. Hypertension was the most common comorbidity observed in patients receiving kidney transplants in this study, and the frequency of this comorbidity was significantly higher in the non-PTDM group than in the PTDM group (non-PTDM vs. PTDM, $64.0 \%$ vs. $47.8 \% ; p<0.007$ ). Infection with cytomegalovirus or hepatitis $C$ virus before transplantation was not significantly related to the development of PTDM. Furthermore, the frequency of recipients of tacrolimus-based IMRs was significantly higher in the PTDM group than in the non-PTDM group (non-PTDM vs. PTDM, 55.0 vs. $69.6 \%$; $p<0.001$ ). 
Table 2. Incidence of PTDM during the follow-up periods $(n=425)$.

\begin{tabular}{ccccccccccc}
\hline Years post KT & $\mathbf{1}$ & $\mathbf{2}$ & $\mathbf{3}$ & $\mathbf{4}$ & $\mathbf{5}$ & $\mathbf{6}$ & $\mathbf{7}$ & $\mathbf{8}$ & $\mathbf{9}$ & $\mathbf{1 0}$ \\
\hline Total patients & 388 & 362 & 335 & 315 & 290 & 262 & 233 & 206 & 185 & 166 \\
Patients with PTDM & 30 & 10 & 10 & 10 & 6 & 6 & 9 & 4 & 2 & 5 \\
Incidence (\%) & 7.73 & 2.76 & 2.99 & 3.17 & 2.07 & 2.29 & 3.86 & 1.94 & 1.08 & 3.01 \\
Cumulative patients & 30 & 40 & 50 & 60 & 66 & 72 & 81 & 85 & 87 & 92 \\
Cumulative incidence (\%) & 7.15 & 9.58 & 12.13 & 14.78 & 16.44 & 18.24 & 21.18 & 22.59 & 23.39 & 25.57 \\
\hline
\end{tabular}

KT: kidney transplantation; PTDM: post-transplant diabetes mellitus.

\subsection{Risk Factors of PTDM}

The multivariable logistic regression analyses revealed that age $(p<0.001)$, BMI $(p<0.001)$, donor source $(p<0.04)$, and tacrolimus use $(p<0.001)$ were independent risk factors for PTDM (Table 3$)$. The risk of developing PTDM in recipients aged 45-65 years at the time of transplant was 2.9 times higher than that in patients aged $<45$ years $(\mathrm{aOR}=2.90,95 \%$ confidence interval $(\mathrm{CI})=1.64-5.13)$, and the risk in patients aged $>65$ years was 4.86 times higher than that in patients aged $<45$ years $(\mathrm{aOR}=4.86$, $95 \% \mathrm{CI}=1.5-15.79)$. The risk of developing PTDM in recipients with BMI $24-27$ and $>27 \mathrm{~kg} / \mathrm{m}^{2}$ was 2.96 times $(\mathrm{aOR}=2.96,95 \% \mathrm{CI}=1.52-5.75)$ and 5.25 times $(\mathrm{aOR}=5.25,95 \% \mathrm{CI}=2.75-10.42)$ higher than that in patients with BMI $<24$, respectively. The risk of developing PTDM in patients who received cadaveric donor renal transplants was 2.01 times higher than that in patients who received living-patient renal transplants $(\mathrm{OR}=2.01,95 \% \mathrm{CI}=1.03-3.93)$. Furthermore, the risk of developing PTDM in recipients of tacrolimus-based IMRs was 3.57 times higher than that in patients receiving the tacrolimus-based + mTORi regimen $(\mathrm{OR}=3.57,95 \% \mathrm{CI}=0.14-0.55)$. Finally, the cumulative incidence of PTDM in recipients of tacrolimus-based IMRs was significantly higher than that in patients receiving the tacrolimus-based + mTORi regimen ( $p<0.001$; Kaplan-Meier analysis; Figure 2).

Table 3. Logistic regression analysis for risk factors for PTDM.

\begin{tabular}{|c|c|c|c|c|}
\hline & \multicolumn{2}{|c|}{ Univariable } & \multicolumn{2}{|c|}{ Multivariable } \\
\hline & OR $(95 \% \mathrm{CI})$ & $p$-Value & OR $(95 \% \mathrm{CI})$ & $p$-Value \\
\hline \multicolumn{5}{|c|}{ Gender (vs. female) } \\
\hline Male & $1.33(0.84-2.13)$ & 0.23 & $1.30(0.76-2.21)$ & 0.33 \\
\hline \multicolumn{5}{|c|}{ Age at KT, years (vs. <45 years) } \\
\hline $45-65$ & $2.62(1.60-4.29)$ & $<0.001 * *$ & $2.90(1.64-5.13)$ & $<0.001 * *$ \\
\hline$>65$ & $3.38(1.17-9.80)$ & $0.03 *$ & $4.86(1.50-15.79)$ & $0.008^{* *}$ \\
\hline \multicolumn{5}{|c|}{ Donor source (vs. living) } \\
\hline Cadaveric & $2.30(1.29-4.07)$ & $0.004^{* *}$ & $2.01(1.03-3.93)$ & $0.040 *$ \\
\hline \multicolumn{5}{|c|}{ BMI, $\mathrm{kg} / \mathrm{m}^{2}$ (vs. BMI <24) } \\
\hline $24-27$ & $2.75(1.53-4.92)$ & $0.001^{* *}$ & $2.96(1.52-5.75)$ & $0.001^{* *}$ \\
\hline$>27$ & $4.15(2.32-7.44)$ & $<0.001^{* *}$ & $5.35(2.75-10.42)$ & $<0.001^{* *}$ \\
\hline \multicolumn{5}{|c|}{ IMR groups (vs. FK-based) } \\
\hline $\mathrm{FK}+\mathrm{mTORi}$ & $0.39(0.22-0.70)$ & $0.002 * *$ & $0.28(0.14-0.55)$ & $<0.001^{* *}$ \\
\hline CsA & $2.14(0.86-5.33)$ & 0.10 & $1.40(0.49-3.97)$ & 0.53 \\
\hline $\mathrm{CsA}+\mathrm{mTORi}$ & $0.41(0.09-1.85)$ & 0.25 & $0.30(0.06-1.64)$ & 0.17 \\
\hline
\end{tabular}

\subsection{Patient and Allograft Survival rates}

A Kaplan-Meier analysis revealed that the 10-year cumulative survival rate of transplant recipients was significantly lower in the PTDM group than in the non-PTDM group ( $p<0.007$; Figure 3A). The 10-year cumulative graft survival rate was also significantly lower in the PTDM group than in the non-PTDM group (Figure 3B). 


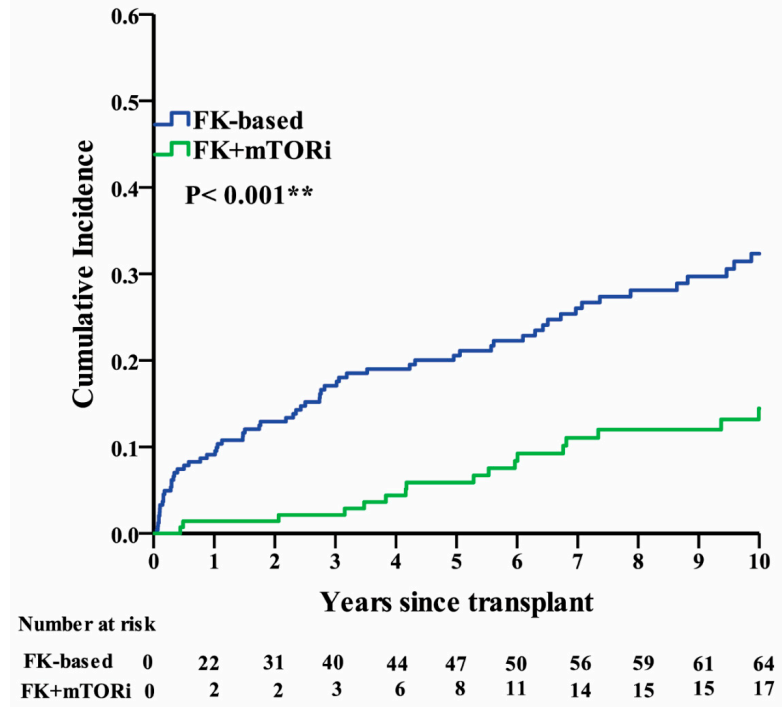

Figure 2. Kaplan-Meier analysis of the cumulative incidence of post-transplant diabetes mellitus (PTDM) in the tacrolimus (FK)-based and FK + mammalian target of rapamycin inhibitor (mTORi)-based regimen groups.
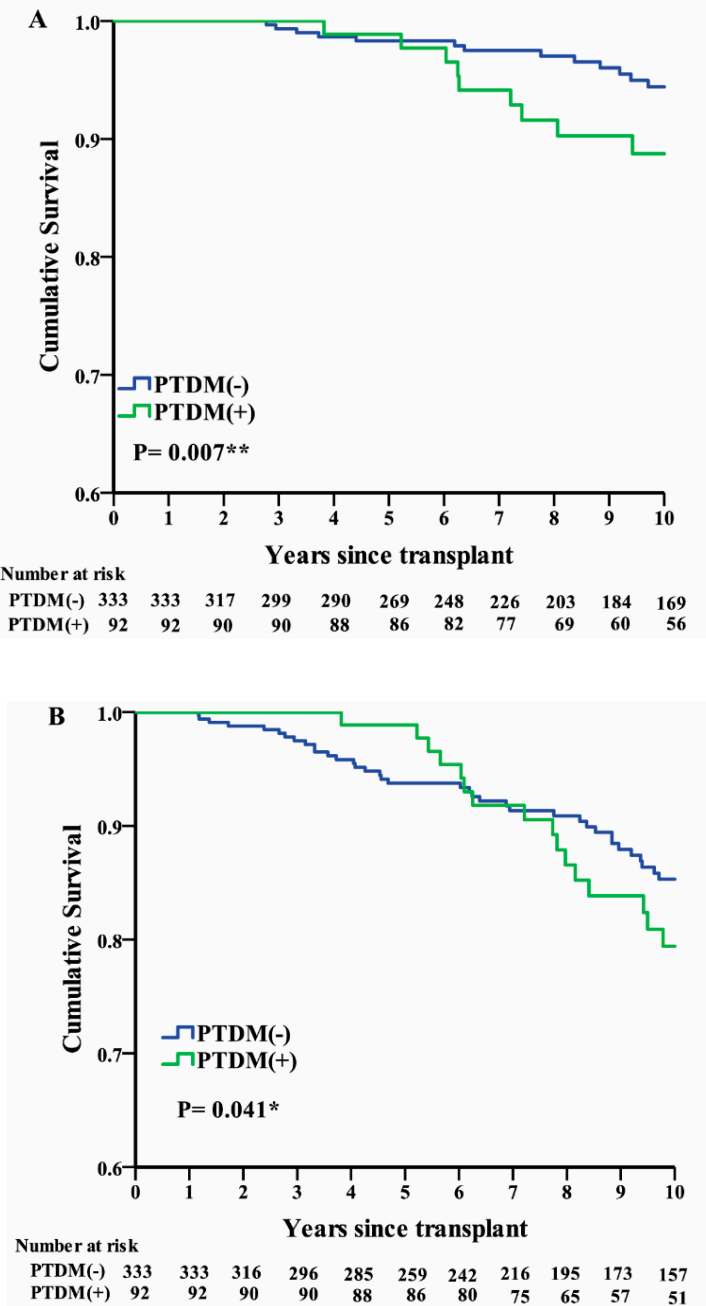

Figure 3. Cumulative survival rates in the post-transplant diabetes mellitus (PTDM) and non-PTDM groups for (A) transplant recipients and (B) kidney allografts. 


\section{Discussion}

\subsection{Incidence of PTDM}

We examined the incidence of and risk factors for PTDM after kidney transplantation in Taiwanese patients, and there have been few such studies in the past. At 1, 3, and 5 years after transplantation, the proportion of patients who developed PTDM (Table 2) was not similar to that reported in previous studies (e.g., 13.00\% (American study) and 11.80\% (Korean study) after 1 year, 30.72\% after 3 years (Chinese study), and 15.10\% after 5 years (Japanese study)) [2,10,16,17]. The incidence rate of PTDM in both the American and Korean studies was higher than that reported here; this could be attributed to the relatively higher BMI in the American study $\left(27 \pm 5 \mathrm{~kg} / \mathrm{m}^{2}\right)$ or the inclusion of patients with temporary hyperglycemia resulting from the consumption of high doses of corticosteroids to prevent acute rejection in the Korean study $[2,16]$. The 3-year cumulative incidence of PTDM in the Chinese study was higher than that reported here, and this can be attributed to the inclusion of patients with temporary hyperglycemia in the Chinese study, as in the Korean study [16,17]. However, the 5-year cumulative incidence rate of PTDM in the Japanese study was lower than that in our study, which may be related to the lower serum tacrolimus concentration in the Japanese study (3.8-7.6 ng/mL). The variability in the incidence of PTDM among these studies is due to the diagnostic criteria for PTDM, therapeutic protocols for post-transplant immunosuppression, duration of follow-up, and study population; therefore, the studies may have some deviations in the results. The main purpose of this study was to investigate the long-term incidence of PTDM after kidney transplantation; consequently, patients with temporary PTDM were excluded. Thus, this is a distinguishing feature of our study.

\subsection{Risk Factors of PTDM}

The following four variables with an effect on PTDM development were identified: age $(p<0.001)$, BMI $(p<0.001)$, donor source $(p<0.004)$, and immunosuppressive agent used $(p=0.001 ;$ Table 1$)$. Using multivariate logistic regression methods, we showed that the risk of developing PTDM in recipients aged $\geq 45$ years was $2.9-4.86$ times higher than that in patients aged $<45$ years, highlighting the correlation between age and PTDM risk [10,16-18].

A high BMI in the context of a transplant may affect transplant outcomes, which has been shown in previous studies [3,19-21]. Our results showed that BMI was positively correlated with the OR of developing PTDM. There were no patients with obesity in this study; thus, the incidence of PTDM was lower than that reported in other studies.

The donor source was an independent risk factor for PTDM, as recipients of deceased donor kidneys had a 1.5-3.7 times higher risk of developing PTDM than those receiving live donor kidneys [19,22]. The ORs of developing PTDM were higher in patients receiving a deceased donor kidney than in patients receiving a live donor organ (Table 3), and this is similar to results previously reported [19,22]. We postulate that this may be related to the greater use of immunosuppressants (i.e., induction therapy, higher levels of CNI, and higher steroid doses) in deceased donor transplant recipients. Although further evidence is required to support the increased risk of developing PTDM associated with deceased donor transplants, deceased donor transplant recipients should be identified as having a higher risk of developing PTDM than live donor recipients, informed, and cautiously monitored [22].

The use of tacrolimus (vs. cyclosporine) is independently associated with PTDM in several studies $[3,22,23]$. However, the incidence of PTDM among recipients of tacrolimus and mTORi concurrently (vs. cyclosporine and mTORi) was considerably lower than that among patients receiving CNI-based regimens (Table 1). Our multivariate logistic regression analyses showed that recipients of cyclosporine-based regimens had a higher risk of developing PTDM than those receiving tacrolimus-based regimens, although this was not statistically significant. Therefore, the diabetogenic effects of both cyclosporine and tacrolimus were comparable (Table 2). Recipients concurrently using tacrolimus and mTORis also had a considerably lower risk of developing PTDM than those receiving 
tacrolimus-based regimens $(p<0.001)$. However, this was in contrast to the results reported by Johnston et al. using the United States Renal Data System registry [24].

The use of CNIs such as cyclosporine and tacrolimus remains the backbone of immunosuppressive treatment for most kidney transplant recipients in our center, and an mTORi may be added to minimize the adverse effects of CNIs and prevent organ rejection. Individualized adjustment of IMRs for kidney transplant recipients helps to maintain graft function and decrease the adverse reactions associated with IMRs, which in turn may protect grafts from the risk of rejection. The diabetogenic effects of tacrolimus are relatively strong and dose-related $[23,25]$. When tacrolimus is administered with an mTORi to prevent acute rejection, both tacrolimus and mTORi may be used at lower doses; thus, the diabetogenic effects of tacrolimus are reduced [26-28]. This may explain why patients receiving concurrent therapy with tacrolimus and mTORi had a lower risk of developing PTDM than those receiving tacrolimus- or cyclosporine-based regimens. In addition, insulin sensitivity does not significantly change when non-obese recipients $\left(\mathrm{BMI}<30 \mathrm{~kg} / \mathrm{m}^{2}\right.$ ) are administered tacrolimus or sirolimus [20]. This may also indirectly explain the lower incidence of PTDM observed in the PTDM group in our study, as the median BMI was $24.6 \mathrm{~kg} / \mathrm{m}^{2}$. A higher prevalence of hypertension was observed in the non-PTDM group than in the PTDM group (Table 1). A meta-analysis study reported that renin-angiotensin system (RAS) inhibitors (angiotensin-converting enzyme inhibitors or angiotensin receptor blockers) reduced the incidence of newly diagnosed diabetes (27\% and $23 \%$, respectively) [29]. Investigation on the use of RAS in our study population showed that patients in the non-PTDM group used more RAS inhibitors than patients in the PTDM group ( $48.6 \%$ vs. $25.0 \%, p<0.001)$. Therefore, it is speculated that the long-term use of RAS inhibitors may protect patients with hypertension before transplantation from PTDM risk. This may also explain why patients with high blood pressure before transplantation have a lower risk of PTDM incidence.

\subsection{Patient and Allograft Survival Rates}

Although studies had shown that the major causes of death in patients with PTDM are related to cardiovascular disease [30,31], the use of immunosuppressants after kidney transplantation can allow severe infection and even death in patients with PTDM [32]. In our study, infection was the main cause of death after transplantation (Table 1), which may explain the lower survival rates after 10 years in patients with PTDM than in patients without PTDM (Figure 3A). Thus, the increased mortality risk in patients with PTDM may have been related to drug-drug interactions and infections associated with immunosuppressant medications. In our study, the allograft survival rate after 10 years was significantly lower in recipients in the PTDM group, which was in accordance with evidence published by Kuo et al. using the Organ Procurement and Transplant Network/United Network for Organ Sharing database [33].

\subsection{Study Limitations}

This study had certain limitations. First, the study population only represented patients receiving kidney transplantations in a single center, indicating that these results should be generalized with caution. Second, tacrolimus was made available in 1998 and became popular because of its effects in preventing acute rejection. Cyclosporine is less frequently prescribed and statistical errors may have occurred because of the differences in patient sample sizes. Finally, only patients who underwent just one transplantation were included for analysis in the study, and those whose treatment was switched between tacrolimus and cyclosporine were excluded $(n=99)$. Thus, it is difficult to extrapolate the frequency of PTDM in patients who received multiple transplants and the correlation between PTDM and changes in tacrolimus/cyclosporine prescription.

\section{Conclusions}

This study revealed that high age and BMI at transplantation and receiving an organ from a deceased donor were the three most important risk factors for developing PTDM. The cumulative 
survival rates of patients and allografts were higher in patients with PTDM than in those without PTDM. Furthermore, concurrent use of CNIs and mTORis may decrease the risk of PTDM. Screening patients to identify PTDM risk factors before transplantation and modifying immunosuppressant regimens after transplantation may effectively prevent the development of PTDM. Further prospective research is needed to understand the relationship between immunosuppressant regimens and PTDM.

Author Contributions: Conceptualization, C.-Y.C. and H.-Y.W.; methodology, C.-Y.C., H.-Y.W., and Y.-M.L.; software and formal analysis, J.-P.C.; data curation C.-Y.C. and Y.-M.L.; writing-original draft preparation, and writing-review and editing, C.-Y.C. and H.-Y.W.; supervision, C.-H.C., M.-F.W., M.-J.W., and Y.-C.H. All authors have read and agreed to the published version of the manuscript.

Funding: This research received no external funding.

Acknowledgments: We express our gratitude to Richard H. Davis, a second-language teacher, for his assistance with English editing.

Conflicts of Interest: The authors declare no conflict of interest.

\section{References}

1. Kasiske, B.L.; Snyder, J.J.; Gilbertson, D.; Matas, A.J. Diabetes mellitus after kidney transplantation in the United States. Am. J. Transplant. 2003, 3, 178-185. [CrossRef]

2. Cosio, F.G.; Kudva, Y.; van der Velde, M.; Larson, T.S.; Textor, S.C.; Griffin, M.D.; Stegall, M.D. New onset hyperglycemia and diabetes are associated with increased cardiovascular risk after kidney transplantation. Kidney Int. 2005, 67, 2415-2421. [CrossRef] [PubMed]

3. Baron, P.W.; Infante, S.; Peters, R.; Tilahun, J.; Weissman, J.; Delgado, L.; Kore, A.H.; Beeson, W.L.; de Vera, M.E. Post-Transplant Diabetes Mellitus after Kidney Transplant in Hispanics and Caucasians Treated with Tacrolimus-Based Immunosuppression. Ann. Transplant. 2017, 22, 309-314. [CrossRef] [PubMed]

4. Cosio, F.G.; Pesavento, T.E.; Osei, K.; Henry, M.L.; Ferguson, R.M. Post-transplant diabetes mellitus: Increasing incidence in renal allograft recipients transplanted in recent years. Kidney Int. 2001, 59, $732-737$. [CrossRef] [PubMed]

5. Eide, I.A.; Halden, T.A.; Hartmann, A.; Asberg, A.; Dahle, D.O.; Reisaeter, A.V.; Jenssen, T. Mortality risk in post-transplantation diabetes mellitus based on glucose and HbA1c diagnostic criteria. Transpl. Int. 2016, 29, 568-578. [CrossRef] [PubMed]

6. Park, S.C.; Yoon, Y.D.; Jung, H.Y.; Kim, K.H.; Choi, J.Y.; Park, S.H.; Kim, C.D.; Kim, Y.L.; Kim, H.K.; Huh, S.; et al. Effect of transient post-transplantation hyperglycemia on the development of diabetes mellitus and transplantation outcomes in kidney transplant recipients. Transplant. Proc. 2015, 47, 666-671. [CrossRef] [PubMed]

7. Davidson, J.; Wilkinson, A.; Dantal, J.; Dotta, F.; Haller, H.; Hernandez, D.; Kasiske, B.L.; Kiberd, B.; Krentz, A.; Legendre, C.; et al. New-onset diabetes after transplantation: 2003 International consensus guidelines. Transplantation 2003, 75, SS3-SS24. [CrossRef]

8. Wilkinson, A.; Davidson, J.; Dotta, F.; Home, P.D.; Keown, P.; Kiberd, B.; Jardine, A.; Levitt, N.; Marchetti, P.; Markell, M.; et al. Guidelines for the treatment and management of new-onset diabetes after transplantation. Clin. Transplant. 2005, 19, 291-298. [CrossRef]

9. Alsheikh, R.; Gabardi, S. Post-Renal Transplantation Outcomes in Elderly Patients Compared to Younger Patients in the Setting of Early Steroid Withdrawal. Prog. Transplant. 2018, 28, 322-329. [CrossRef]

10. Okumi, M.; Unagami, K.; Hirai, T.; Shimizu, T.; Ishida, H.; Tanabe, K. Diabetes mellitus after kidney transplantation in Japanese patients: The Japan Academic Consortium of Kidney Transplantation study. Int. J. Urol. Off. J. Jpn. Urol. Assoc. 2017, 24, 197-204. [CrossRef]

11. Tomkins, M.; Tudor, R.M.; Cronin, K.; O'Kelly, P.; Williams, Y.; Little, D.; de Freitas, D.G.; Denton, M.; O'Seaghdha, C.; Conlon, P.; et al. Risk factors and long-term consequences of new-onset diabetes after renal transplantation. Ir. J. Med. Sci. 2020, 189, 497-503. [CrossRef] [PubMed]

12. Jenssen, T.; Hartmann, A. Emerging treatments for post-transplantation diabetes mellitus. Nat. Rev. Nephrol. 2015, 11, 465-477. [CrossRef] [PubMed] 
13. Tarnowski, M.; Sluczanowska-Glabowska, S.; Pawlik, A.; Mazurek-Mochol, M.; Dembowska, E. Genetic factors in pathogenesis of diabetes mellitus after kidney transplantation. Ther. Clin. Risk Manag. 2017, 13, 439-446. [CrossRef] [PubMed]

14. Montero, N.; Pascual, J. Immunosuppression and Post-transplant Hyperglycemia. Curr. Diabetes Rev. 2015, 11, 144-154. [CrossRef]

15. Sharif, A.; Hecking, M.; de Vries, A.P.; Porrini, E.; Hornum, M.; Rasoul-Rockenschaub, S.; Berlakovich, G.; Krebs, M.; Kautzky-Willer, A.; Schernthaner, G.; et al. Proceedings from an international consensus meeting on posttransplantation diabetes mellitus: Recommendations and future directions. Am. J. Transplant. 2014, 14, 1992-2000. [CrossRef]

16. Paek, J.H.; Kang, S.S.; Park, W.Y.; Jin, K.; Park, S.B.; Han, S.; Kim, C.D.; Ro, H.; Lee, S.; Jung, C.W.; et al. Incidence of Post-transplantation Diabetes Mellitus Within 1 Year after Kidney Transplantation and Related Factors in Korean Cohort Study. Transplant. Proc. 2019, 51, 2714-2717. [CrossRef]

17. Xu, J.; Xu, L.; Wei, X.; Li, X.; Cai, M. Incidence and Risk Factors of Posttransplantation Diabetes Mellitus in Living Donor Kidney Transplantation: A Single-Center Retrospective Study in China. Transplant. Proc. 2018, 50, 3381-3385. [CrossRef]

18. Sinangil, A.; Celik, V.; Barlas, S.; Koc, Y.; Basturk, T.; Sakaci, T.; Akin, E.B.; Ecder, T. The incidence of new onset diabetes after transplantation and related factors: Single center experience. Nefrologia 2017, 37, 181-188. [CrossRef]

19. Lv, C.; Chen, M.; Xu, M.; Xu, G.; Zhang, Y.; He, S.; Xue, M.; Gao, J.; Yu, M.; Gao, X.; et al. Influencing factors of new-onset diabetes after a renal transplant and their effects on complications and survival rate. PLoS ONE 2014, 9, e99406. [CrossRef]

20. Cascais de Sa, D.; Santos, L.; Rodrigues, L.; Romaozinho, C.; Guedes Marques, M.; Macario, F.; Bastos, M.; Baptista, C.; Alves, R.; Figueiredo, A. Post-Kidney-Transplant Diabetes: Evaluation of Risk Factors. Transplant. Proc. 2019, 51, 1597-1600. [CrossRef]

21. Jensen, M.D.; Ryan, D.H.; Apovian, C.M.; Ard, J.D.; Comuzzie, A.G.; Donato, K.A.; Hu, F.B.; Hubbard, V.S.; Jakicic, J.M.; Kushner, R.F.; et al. 2013 AHA/ACC/TOS guideline for the management of overweight and obesity in adults: A report of the American College of Cardiology/American Heart Association Task Force on Practice Guidelines and The Obesity Society. Circulation 2014, 129, S102-S138. [CrossRef] [PubMed]

22. Gourishankar, S.; Jhangri, G.S.; Tonelli, M.; Wales, L.H.; Cockfield, S.M. Development of diabetes mellitus following kidney transplantation: A Canadian experience. Am. J. Transplant. 2004, 4, 1876-1882. [CrossRef] [PubMed]

23. Veroux, M.; Tallarita, T.; Corona, D.; Sinagra, N.; Giaquinta, A.; Zerbo, D.; Guerrieri, C.; D'Assoro, A.; Cimino, S.; Veroux, P. Conversion to sirolimus therapy in kidney transplant recipients with new onset diabetes mellitus after transplantation. Clin. Dev. Immunol. 2013, 2013, 496974. [CrossRef] [PubMed]

24. Johnston, O.; Rose, C.L.; Webster, A.C.; Gill, J.S. Sirolimus is associated with new-onset diabetes in kidney transplant recipients. J. Am. Soc. Nephrol. 2008, 19, 1411-1418. [CrossRef]

25. Webster, A.C.; Woodroffe, R.C.; Taylor, R.S.; Chapman, J.R.; Craig, J.C. Tacrolimus versus ciclosporin as primary immunosuppression for kidney transplant recipients: Meta-analysis and meta-regression of randomised trial data. BMJ (Clin. Res. Ed.) 2005, 331, 810. [CrossRef]

26. Undre, N.A. Pharmacokinetics of tacrolimus-based combination therapies. Nephrol. Dial. Transplant. 2003, 18 (Suppl. 1), i12-i15. [CrossRef]

27. Pascual, J.; del Castillo, D.; Cabello, M.; Pallardo, L.; Grinyo, J.M.; Fernandez, A.M.; Brunet, M. Interaction between everolimus and tacrolimus in renal transplant recipients: A pharmacokinetic controlled trial. Transplantation 2010, 89, 994-1000. [CrossRef]

28. Larsen, J.L.; Bennett, R.G.; Burkman, T.; Ramirez, A.L.; Yamamoto, S.; Gulizia, J.; Radio, S.; Hamel, F.G. Tacrolimus and sirolimus cause insulin resistance in normal sprague dawley rats. Transplantation 2006, 82, 466-470. [CrossRef]

29. Abuissa, H.; Jones, P.G.; Marso, S.P.; O’Keefe, J.H., Jr. Angiotensin-converting Enzyme Inhibitors or Angiotensin Receptor Blockers for Prevention of Type 2 Diabetes: A Meta-Analysis of Randomized Clinical Trials. J. Am. Coll. Cardiol. 2005, 46, 821-826. [CrossRef]

30. Li, S.; Wang, J.; Zhang, B.; Li, X.; Liu, Y. Diabetes Mellitus and Cause-Specific Mortality: A Population-Based Study. Diabetes Metab. J. 2019, 43, 319-341. [CrossRef] 
31. Wauters, R.P.; Cosio, F.G.; Suarez Fernandez, M.L.; Kudva, Y.; Shah, P.; Torres, V.E. Cardiovascular consequences of new-onset hyperglycemia after kidney transplantation. Transplantation 2012, 94, 377-382. [CrossRef] [PubMed]

32. Shivaswamy, V.; Boerner, B.; Larsen, J. Post-Transplant Diabetes Mellitus: Causes, Treatment, and Impact on Outcomes. Endocr. Rev. 2016, 37, 37-61. [CrossRef] [PubMed]

33. Kuo, H.T.; Sampaio, M.S.; Vincenti, F.; Bunnapradist, S. Associations of pretransplant diabetes mellitus, new-onset diabetes after transplant, and acute rejection with transplant outcomes: An analysis of the Organ Procurement and Transplant Network/United Network for Organ Sharing (OPTN/UNOS) database. Am. J. Kidney Dis. 2010, 56, 1127-1139. [CrossRef] [PubMed]

(C) 2020 by the authors. Licensee MDPI, Basel, Switzerland. This article is an open access article distributed under the terms and conditions of the Creative Commons Attribution (CC BY) license (http://creativecommons.org/licenses/by/4.0/). 